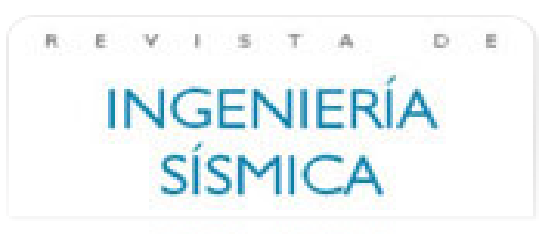

\section{Revista de Ingeniería Sísmica}

ISSN: 0185-092X

smis@smis.org.mx

Sociedad Mexicana de Ingeniería

Sísmica

México

Tapia Hernández, Edgar; Tena Colunga, Arturo

DISEÑO SÍSMICO DE MARCOS DE ACERO CONTRAVENTEADOS. PARTE 2: EVALUACIÓN DE LA METODOLOGÍA

Revista de Ingeniería Sísmica, núm. 88, 2013, pp. 69-90

Sociedad Mexicana de Ingeniería Sísmica

Distrito Federal, México

Disponible en: http://www.redalyc.org/articulo.oa?id=61827446005

- Cómo citar el artículo

- Número completo

- Más información del artículo

- Página de la revista en redalyc.org

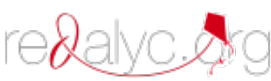

Sistema de Información Científica

Red de Revistas Científicas de América Latina, el Caribe, España y Portugal

Proyecto académico sin fines de lucro, desarrollado bajo la iniciativa de acceso abierto 


\title{
DISEÑO SÍSMICO DE MARCOS DE ACERO CONTRAVENTEADOS. PARTE 2: EVALUACIÓN DE LA METODOLOGÍA
}

\author{
Edgar Tapia Hernández ${ }^{(1)}$ y Arturo Tena Colunga ${ }^{(2)}$
}

\begin{abstract}
RESUMEN
Se presentan los resultados de un estudio de seis edificios regulares de 10, 14 y 18 pisos estructurados con marcos de acero dúctiles con contraventeo concéntrico. Los modelos, que se diseñaron por capacidad siguiendo una metodología propuesta para este sistema estructural, se ubicaron en la subzona IIIa del Reglamento de Construcciones para el Distrito Federal (RCDF-04). Los análisis no lineales se realizaron en OpenSees considerando la sobrerresistencia del material en el comportamiento de los edificios con base en 290 certificados de laboratorio de perfiles disponibles en el mercado mexicano.
\end{abstract}

Palabras clave: marcos dúctiles, contraventeo concéntrico, diseño por capacidad, ductilidad, factor de reducción por sobrerresistencia, desempeño estructural.

\begin{abstract}
Results related to the study of six regular steel buildings structured with ductile concentrically braced frames of 10-, 14- and 18-stories are reported. Models were designed following a proposed capacity methodology for this structural system and were located in subzone IIIa of the Mexico's Federal District Code. Nonlinear analyses were performed in OpenSees considering the material overstrength through 292 certified laboratory coupon tests of steel samples available in the Mexican market.
\end{abstract}

Keywords: ductile frames, concentric bracing, capacity design, ductility, overstrength factor, structural performance.

\section{INTRODUCCIÓN}

Los resultados de estudios recientes (Tapia 2005, Lacerte y Tremblay 2006, Ozgur 2006, Izvernari 2007, Tapia y Tena 2011) demuestran que los edificios estructurados con marcos de acero contraventeados diseñados racionalmente siguiendo los criterios de la reglamentación vigente, no desarrollan necesariamente resultados congruentes con las premisas de diseño donde el mecanismo último de colapso debe corresponder a columna fuerte - viga débil - contraviento más débil, sino que se presentan mecanismos de colapso mixtos, con articulación de columnas que favorecen la formación de pisos débiles, reservas considerables de sobrerresistencia, redistribuciones de cargas imprevistas, etc.

Artículo recibido el 4 de mayo de 2012 y aprobado para su publicación el 9 de enero de 2013. Se aceptarán comentarios y/o discusiones hasta cinco meses después de su publicación.

(1) Profesor, Departamento de Materiales, Universidad Autónoma Metropolitana Azcapotzalco, Av. San Pablo 180, Col. Reynosa Tamaulipas, 02200 México, DF, e-mail: etapiah@correo.azc.uam.mx

(2) Profesor, Departamento de Materiales, Universidad Autónoma Metropolitana Azcapotzalco, Av. San Pablo 180, Col. Reynosa Tamaulipas, 02200 México, DF, e-mail: atc@correo.azc.uam.mx 
Estudios previos llevados a cabo por los autores (Tapia y Tena 2011), aunados a los lineamientos de reglamentos internacionales especializados, resultados de pruebas experimentales y otras investigaciones recientes sirvieron de fundamento para desarrollar una metodología de diseño por capacidad adaptada al Reglamento de Construcciones para el Distrito Federal, que pretende establecer los requisitos mínimos que deben ser considerados en el diseño de este sistema estructural para que los mecanismos de colapso desarrollados concuerden con las premisas implícitas en el diseño (columna fuerte - viga débil contraviento más débil), con una adecuada distribución de la respuesta inelástica en la altura y redistribuciones de cargas predecibles. Es decir, la metodología propuesta pretende que, ante una demanda sísmica intensa, la disipación de la energía se realice con daño controlado en los contravientos y también en las trabes, pero con poco o nulo daño en las columnas, con el fin de salvaguardar la integridad de los ocupantes y/o los contenidos.

En el artículo complementario, Tapia y Tena (2013) se ejemplifica la metodología de diseño en un modelo de 14 niveles, que forma parte de un conjunto de edificios estructurados con marcos dúctiles de acero contraventeados situados en suelo blando, con alturas entre los 10 a los 18 pisos (35 m a $63 \mathrm{~m}$ ), que se usaron para evaluar la propuesta y cuyos resultados se presentan en este artículo.

\section{DESCRIPCIÓN DE LOS MODELOS}

Se diseñaron tres edificios regulares estructurados con marcos dúctiles de acero con contraventeo concéntrico de 10, 14 y 18 niveles (Ch10_56, Ch14_61 y Ch18_69), cuya configuración se presenta en la figura 1, ubicados en suelo blando (zona IIIa) siguiendo los criterios del Reglamento de Construcciones para el Distrito Federal (RCDF-04) y una propuesta de diseño por capacidad para estos sistemas estructurales.

a)

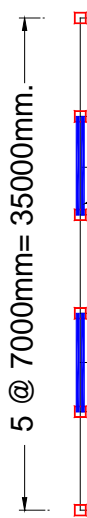

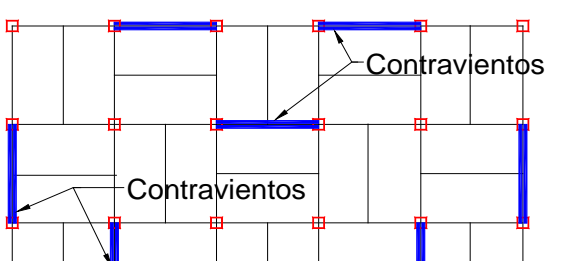

b)
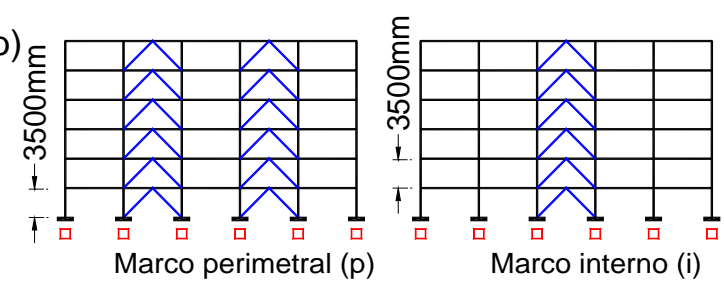

Figura 1. a) Planta tipo, b) Elevación tipo de los marcos estudiados, c) Isométrico del modelo en el Etabs

La metodología propuesta estipula que el diseño de los elementos debe seguir un criterio acorde con el mecanismo de colapso supuesto en la etapa de diseño (columna fuerte - viga débil - contraviento más débil). Las Normas Técnicas Complementarias (NTCS-04) solicitan que al menos el 50\% del cortante lateral sea resistido por las columnas del marco en relación al sistema de contravientos; sin embargo, los resultados de investigaciones conducidas por los autores (Tapia y Tena 2007, 2011) demuestran la 
necesidad de aumentar la aportación de las columnas con el aumento de la esbeltez de los modelos para obtener mecanismos dúctiles siguiendo la ecuación 1. Por esta razón, en la tabla 1, donde se reporta la aportación mínima al cortante que deben resistir los modelos estudiados siguiendo la metodología evaluada, crece con el aumento de la esbeltez $(H / B)$.

$\frac{V_{R C o l}}{V_{\text {Tot }}}=0.50+0.05 \frac{H}{B} \sqrt{\frac{F_{y C o l}}{F_{y \text { Diag }}}}$

donde $H$ es la altura del edificio, $B$ es la dimensión en planta en la dirección de interés $F_{y}$ Diag es el esfuerzo de fluencia de los contravientos y $F_{y}$ Col es el esfuerzo de fluencia de las columnas (Tapia y Tena 2013).

Los factores de reducción en el análisis sísmico de los modelos se establecieron con criterios específicos para este sistema, siguiendo los resultados de investigaciones previas (Tapia y Tena 2011 y Tapia 2011). El factor de comportamiento no lineal $Q$ se definió con la ecuación 2, que asocia el aumento de altura de los modelos con la disminución de la ductilidad que se puede desarrollar, como se explica en el artículo complementario (Tapia y Tena 2013). La obtención del factor de comportamiento sísmico con fines de diseño, $Q$ ', se realizó siguiendo el planteamiento del Apéndice A (NTCS-04), cuyas magnitudes se reportan en la tabla 1.

Tabla 1. Características de los modelos en estudio

\begin{tabular}{lcccccc}
\hline Modelo & $\begin{array}{c}\text { Periodo } \\
\text { (seg) }\end{array}$ & $\begin{array}{c}\text { Relación } \\
\text { H/B }\end{array}$ & $\begin{array}{c}\text { Aportación mínima } \\
\text { al cortante de las } \\
\text { columnas (ec. 1) }\end{array}$ & $\begin{array}{c}\text { Factor de } \\
\text { comportamiento } \\
\text { sísmico Q (ec. 2) }\end{array}$ & $\begin{array}{c}\text { Factor de } \\
\text { comportamiento } \\
\text { sísmico reducido } \\
Q^{\prime} \text { (NTCS) }\end{array}$ & $\begin{array}{c}\text { Factor por } \\
\text { sobrerresistencia } \\
\text { (ec. 3) }\end{array}$ \\
\hline Ch10_56 & 0.721 & 1.00 & $55.9 \%$ & 3.00 & 3.052 & 4.50 \\
Ch14_61 & 1.208 & 1.40 & $61.6 \%$ & 2.33 & 2.368 & 4.50 \\
Ch18_69 & 1.590 & 1.80 & $69.1 \%$ & 2.00 & 2.030 & 4.50 \\
\hline
\end{tabular}

Si $\left\{\begin{array}{cr}\frac{H}{B} \leq 1 & Q=3 \\ 1<\frac{H}{B} \leq 1.6 & Q=3-\frac{5}{3}\left(\frac{H}{B}-1\right) \\ 1.6<\frac{H}{B} & Q=2\end{array}\right.$

El factor de sobrerresistencia $R$ se definió con la ecuación 3, cuya determinación y discusión se puede consultar en Tapia y Tena (2011) y Tapia (2011). La sobrerresistencia asociada a los tres modelos resultó ser igual a la máxima posible (tabla 1) considerando que los modelos se supusieron desplantados en terreno con un periodo fundamental $T_{g}=1.05 \mathrm{seg}$ de la subzona IIIa, por lo que $T_{a}=0.558 \mathrm{seg}$. En la ecuación para marcos dúctiles de acero con contraventeo concéntrico $R_{0}=4.5$ y $\alpha=1.0$ (Tapia y Tena 2013).

$\left.\begin{array}{ll}\text { Si } T \leq T_{a} & R=R_{0}+\alpha\left(1-\sqrt{\frac{T}{T_{a}}}\right) \\ \text { Si } T>T_{a} & R=R_{0}\end{array}\right\}$ 
En el artículo complementario (Tapia y Tena 2013) se detalla el procedimiento de diseño mencionado y las magnitudes reportadas en la tabla 1 del modelo de 14 niveles, un procedimiento de prediseño y las combinaciones de carga consideradas: la combinación de cargas verticales y ocho combinaciones laterales (acción del sismo en dos direcciones ortogonales) con cargas verticales. Además, se ejemplifican las relaciones de esfuerzo obtenidas por las columnas, trabes y contravientos en la combinación que rigió el diseño, donde congruente con un procedimiento de diseño por capacidad, primero se diseñó lo más óptimo posible los elementos que deben fallar primero ante carga lateral (en este caso, contravientos), después las vigas y finalmente las columnas.

\section{SOBRERRESISTENCIA DEL MATERIAL}

Con el objetivo de valorar con mayor certidumbre la influencia del esfuerzo de fluencia real de acero comerciales en México en la sobrerresistencias que puedan desarrollarse, se llevó a cabo un estudio estadístico de los esfuerzos reportados en los certificados de calidad de laboratorios a perfiles laminados rolados en frío (tubos y vigas I) y placas de acero de espesores entre 3/16” (calibre 11, esto es, $0.476 \mathrm{~cm}$ ) hasta 2.5” (6.35 cm). Los certificados de calidad fueron expedidos por distintos laboratorios de perfiles distribuidos en el Distrito Federal y se centran en reportar información de análisis químicos, información mecánica y física (esfuerzo de fluencia, esfuerzo máximo, elongación y la norma de fabricación) y los datos generales (número de colada, embarque, peso y longitud de la pieza). Se hace notar que no es común que estos certificados incluyan otras características del material como la tenacidad, por lo que los resultados presentados están basados en los esfuerzos únicamente.

\section{Acero tipo A - 36}

Se consideraron un total de 260 certificados de acero tipo A - 36, de los cuales el 92.3 por ciento eran de fabricantes nacionales y un 7.7 por ciento provenían de fabricantes extranjeros (China y Estados Unidos). El esfuerzo de fluencia promedio resultó ser igual a $f_{y}=3,264 \mathrm{~kg} / \mathrm{cm}^{2}$, que representa un factor 1.29 veces más grande que el esfuerzo de fluencia teórico $\left(f_{y}=2,530 \mathrm{~kg} / \mathrm{cm}^{2}\right)$. El menor esfuerzo de fluencia se presentó en una placa de 5/16” $(0.79 \mathrm{~cm})$ fabricada en Estados Unidos con $f_{y \text { min }}=2,559$ $\mathrm{kg} / \mathrm{cm}^{2}$, mientras que el mayor esfuerzo de fluencia se reportó en una placa de $3 / 8$ ” $(0.95 \mathrm{~cm})$ fabricada en México con una magnitud de $f_{y \max }=4,675 \mathrm{~kg} / \mathrm{cm}^{2}$.

Por otra parte, el esfuerzo último promedio fue igual a $f_{u}=4,828 \mathrm{~kg} / \mathrm{cm}^{2}$, que representa un factor 1.18 veces mayor al esfuerzo último teórico considerando $f_{u}=4,100 \mathrm{~kg} / \mathrm{cm}^{2}$. En la figura 2 se presenta el esfuerzo de fluencia y el esfuerzo último reportados en los certificados normalizados con los esfuerzos teóricos.

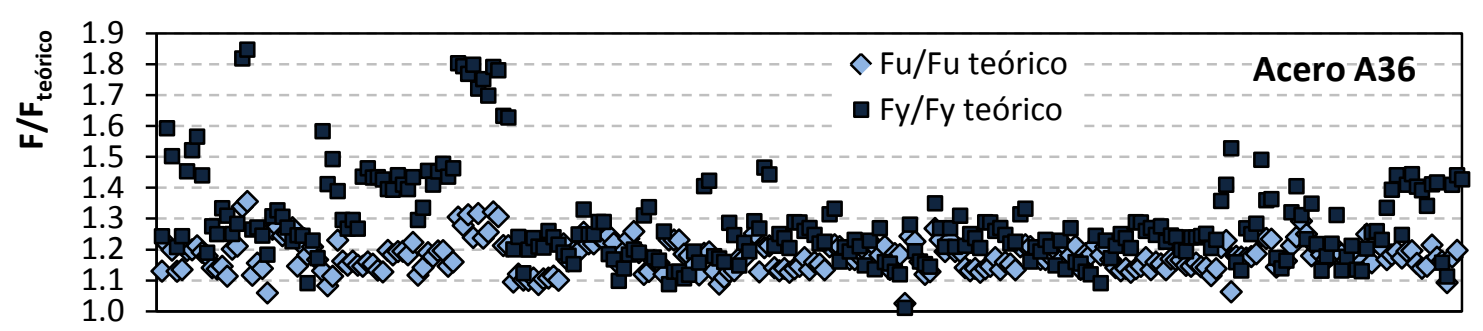

Figura 2. Proporción entre el esfuerzo real y el esfuerzo teórico en placas de acero A-36 


\section{Acero tipo A 572 Gr. 50}

Asimismo, se consideraron 32 certificados de acero tipo A572 Gr. 50, de los cuales el 71.43 por ciento eran de procedencia nacional y un 28.6 por ciento que provenían de fabricantes extranjeros (China y Estados Unidos). El esfuerzo de fluencia promedio resultó ser igual a $f_{y}=4,339 \mathrm{~kg} / \mathrm{cm}^{2}$, que representa un factor 1.23 veces más grande que el esfuerzo de fluencia teórico $\left(f_{y}=3,515 \mathrm{~kg} / \mathrm{cm}^{2}\right)$. El menor esfuerzo de fluencia se presentó en una placa de $1 / 2$ " $(1.27 \mathrm{~cm})$ fabricada en China con $f_{y \text { min }}=4,339 \mathrm{~kg} / \mathrm{cm}^{2}$, mientras que el mayor esfuerzo de fluencia se reportó en una placa de 3/8” $(0.95 \mathrm{~cm})$ fabricada en México, $\operatorname{con} f_{y \max }=4,552 \mathrm{~kg} / \mathrm{cm}^{2}$.

El esfuerzo último promedio fue igual a $f_{u}=5,211 \mathrm{~kg} / \mathrm{cm}^{2}$, que representa un factor 1.14 veces mayor al esfuerzo último teórico considerando $f_{u}=4,570 \mathrm{~kg} / \mathrm{cm}^{2}$. En la figura 3 se presenta el esfuerzo de fluencia y el esfuerzo último reportados en los certificados normalizados con los esfuerzos teóricos.

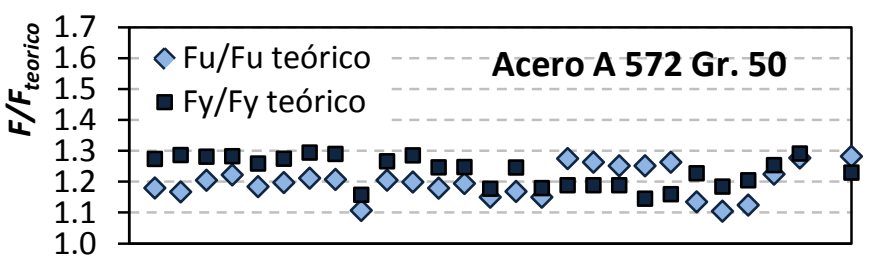

Figura 3. Proporción entre el esfuerzo real y el esfuerzo teórico en placas de acero A-572 Gr. 50

Con base en estos resultados, se realizaron análisis no lineales a los modelos de 10, 14 y 18 pisos en dos escenarios: 1) un análisis considerando los esfuerzos de fluencia teóricos de los aceros $\left(f_{y 36}=2,530\right.$ $\mathrm{kg} / \mathrm{cm}^{2}$ y $f_{\text {y } 50}=3,515 \mathrm{~kg} / \mathrm{cm}^{2}$ ) y, 2) otro con los esfuerzos de fluencia afectados por un factor de amplificación para evaluar la respuesta de la estructura considerando la sobrerresistencia promedio del material $\left(f_{y 36}=3,264 \mathrm{~kg} / \mathrm{cm}^{2}\right.$ y $\left.f_{y 50}=4,339 \mathrm{~kg} / \mathrm{cm}^{2}\right)$. Los análisis que consideran los esfuerzos aumentados (con la sobrerresistencia del material) se distinguieron con una letra a (Ch10_56a, Ch14_61a y Ch18_69a), para diferenciarlos de los análisis con los esfuerzos nominales (Ch10_56, Ch14_61 y Ch18_69). En Tapia (2011) es posible consultar otras fuentes de sobrerresistencia en estructuras metálicas como: elección del tamaño de la sección, sobrerresistencia nominal, esfuerzos de fluencia, endurecimiento por deformación, formación del mecanismo, diseño de conexiones y la influencia de las cargas verticales en el diseño.

Es valioso hacer notar que los 292 certificados de calidad considerados en este estudio, aunque establecen un precedente, no representan un fundamento suficiente para recomendar un aumento en el esfuerzo de fluencia $F_{y}$ que debe considerarse en alguna norma para determinar las capacidades resistentes de los elementos de acero. En cambio, estos resultados son útiles para determinar la resistencia final promedio que podrían desarrollar edificaciones estructuradas con elementos de acero en su conjunto con mayor certidumbre, a través del factor de reducción por sobrerresistencia $R$.

En la figura 4 se muestran los criterios para la determinación del factor por sobrerresistencia conforme al documento ATC-63 (2008) en análisis estáticos ante cargas monótonas crecientes, que se emplearon en esta investigación. En los análisis realizados, los modelos que consideraban la sobrerresistencia del material lograron desarrollar capacidades resistentes y de deformación superiores a los modelos que consideraban la resistencia nominal. 


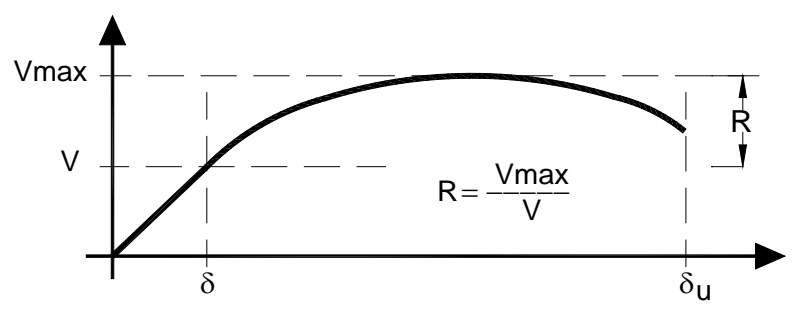

Figura 4. Definición del factor de sobrerresistencia

\section{DETALLES DEL MODELADO}

Los modelos se analizaron en OpenSees (Mazzoni et al. 2006) con el material Steel02 desarrollado con el modelo Giuffre - Menegotto - Pinto, que incluye endurecimiento isotrópico por deformación. La longitud de los contravientos se subdividió en ocho elementos de sección transversal discretizada en fibras de longitud finita para modelar la variación de la deformación a lo largo de cada miembro como se ilustra en la figura 5. En su dimensionamiento se tomaron en cuenta las disminuciones de la longitud efectiva $L_{\text {teorica }} / L_{\text {ejes }}$ descritas detalladamente en la primera parte de esta investigación (Tapia y Tena 2013) y los resultados de investigaciones recientes para determinar la influencia de la subdivisión de los elementos que conforman el contraviento (Izvernari 2007). En Izvernari (2007) se realizó un estudio paramétrico sobre la influencia de la subdivisión de los elementos que conforman el contraviento variando el número de subelementos para el modelado del contraviento en 2, 4, 8, 16, 24 y 32 subdivisiones, considerando la duración del análisis y la variación de los resultados relativos.

Las vigas de las crujías no contraventeadas se modelaron usando el elemento con articulaciones en los extremos (BeamWithHinges), en el que la plasticidad se concentra en la longitud de la articulación definida en los extremos del elemento, con una respuesta elástica en el segmento central. Las vigas de las crujías contraventeadas se dividieron en fibras con forma cuadrilátera con un elemento (NonlinearBeamColumn) en el que la plasticidad se distribuye a lo largo del elemento.

Las columnas se modelaron dividiendo el miembro en ocho subelementos con el comando NonlinearBeamColumn. El modelo analítico propuesto se calibró con resultados de pruebas experimentales a marcos contraventeados (Izvernari 2007).

a)

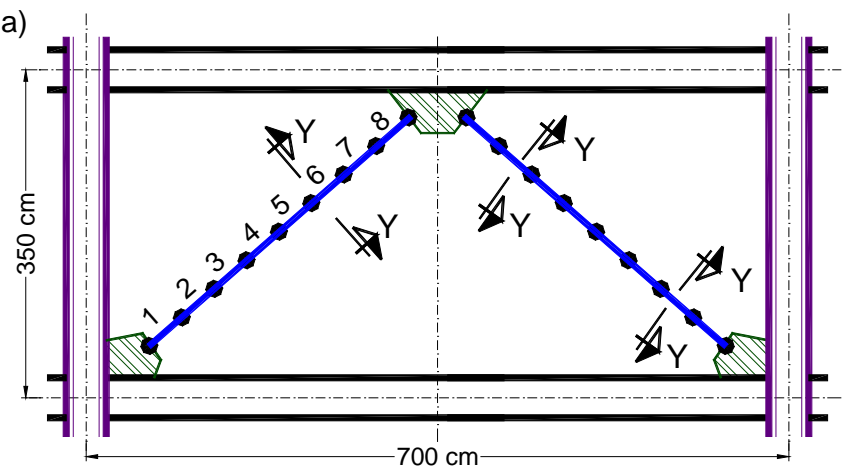

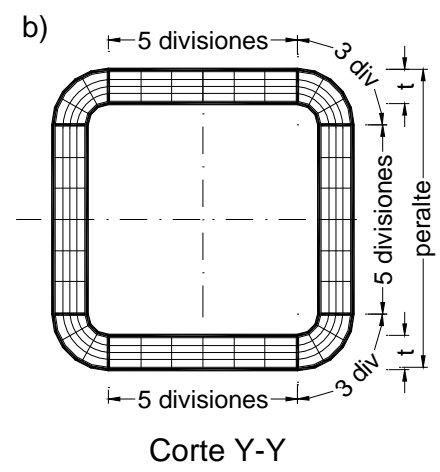

Figura 5. Discretización de la sección transversal y longitudinal de los contravientos 
Adicionalmente, se diseñaron las placas de conexión que se incluyeron en el modelo como resortes en los extremos de los contravientos. En el diseño por compresión de la placa se consideró la sección Whitmore y de las posibles longitudes de pandeo que dependen de las características geométricas.

\section{ANÁLISIS ESTÁTICOS NO LINEALES}

En la figura 6 se presenta la evolución de las distorsiones a lo largo de los análisis al 50 percentil, el 84 percentil y al final del análisis (100 percentil) para evaluar la variabilidad de las deformaciones de entrepiso de los análisis no lineales desarrollados en OpenSees (Mazzoni et al. 2006). El último paso o punto válido del análisis pushover se definió considerando las rotaciones reales y los acortamientos que pueden resistir las secciones estructurales conforme al procedimiento propuesto en Kemp (1996). El modelo propuesto por Kemp (1996) se usa para estimar el momento y la rotación inelástica máxima que puede desarrollar la sección antes de que decrezca en la falla, a partir de la relación de esbeltez de pandeo lateral, factores de esbeltez del pandeo local en los patines y el alma del elemento y un factor de restricción de distorsión.
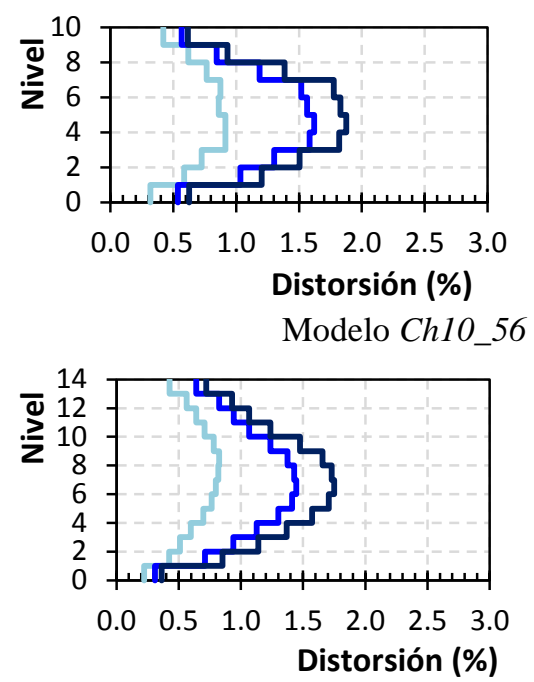

Modelo Ch14_61

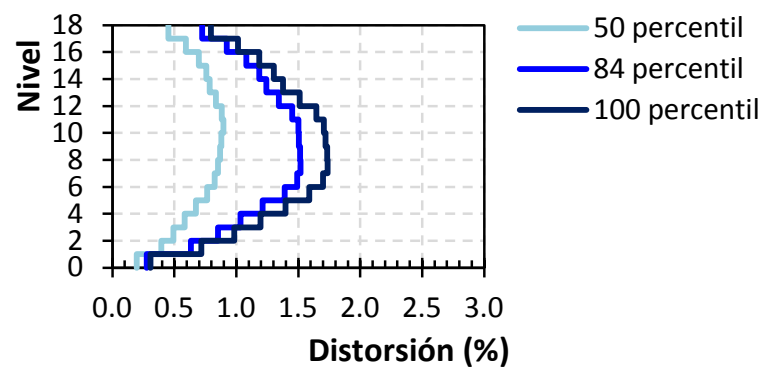

Modelo Ch18 69
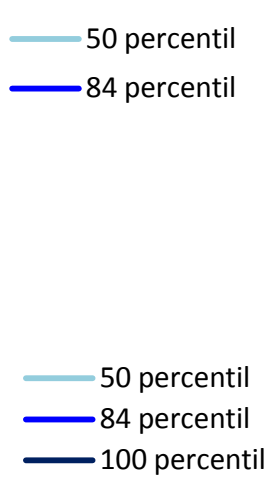

100 percentil

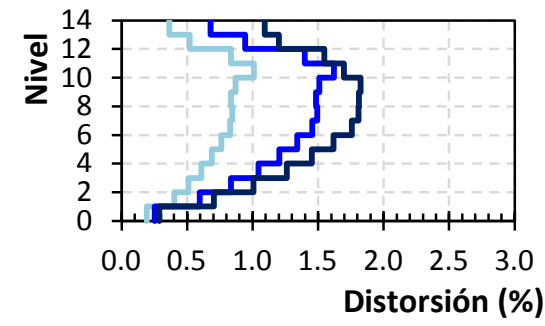

Modelo Ch14_61a

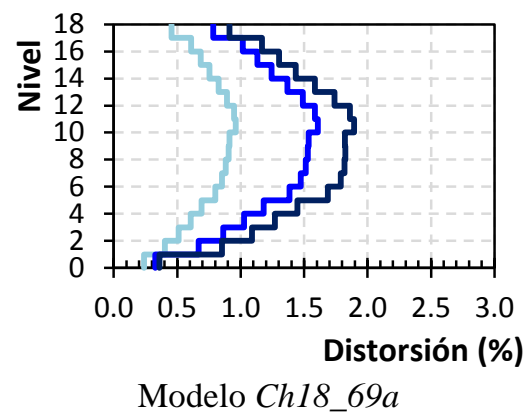

Modelo Ch18_69a

Figura 6. Evolución de las distorsiones (\%) en los modelos de 10, 14 y 18 niveles 
De esta manera, la magnitud máxima reportada en esta investigación no está relacionada con la magnitud del último paso del análisis pushover que el programa calculó, sino con la capacidad máxima de los elementos. El procedimiento detallado se puede consultar en Tapia (2005 y 2011).

Los modelos que incluyen la sobrerresistencia del material tienen la misma configuración deformada que los modelos con el esfuerzo de fluencia teórico. Esa tendencia se presenta desde la mitad de los análisis (50 percentil) con magnitudes de deformación máximas en los pisos intermedios. El límite de distorsión propuesto en las Normas Técnicas (1.5\%) es cercano a la magnitud máxima que reportan las distorsiones en el 84 percentil de los modelos con resistencia del material nominal (Ch10_56, Ch14_61 y Ch18_64), pero este límite se ve superado en los pisos intermedios si se considera la sobrerresistencia en el esfuerzo de fluencia del acero (Ch10_56a, Ch14_61a y Ch18_64a).

\section{Factor de concentración de distorsión}

Se determinó el factor de concentración de distorsión ( $D C F$ por sus siglas en inglés, MacRae et al 2004, Izvernari 2007) para estudiar la tendencia a la formación de pisos débiles, que corresponde a la relación entre la distorsión máxima del entrepiso $\Delta_{i} / h$ y la distorsión global definida como deformación en el último piso $\Delta_{t}$ entre la altura total del edificio $H$ (ecuación 4) como se ilustra en la figura 7.

$D C F=\frac{\Delta_{i} / h}{\Delta_{t} / H}$

Este parámetro se emplea para evaluar la capacidad de la estructura para mitigar la formación de pisos débiles, como indicador de la distribución de la demanda en la altura. Una magnitud máxima del factor de concentración de distorsión menor a $D C F<1.0$ indica que las deformaciones de entrepiso fueron uniformes (no hay formación de pisos débiles), mientras que un factor de concentración de distorsión máximo $D C F>1.0$, refleja concentraciones de demanda en la altura de la estructura.

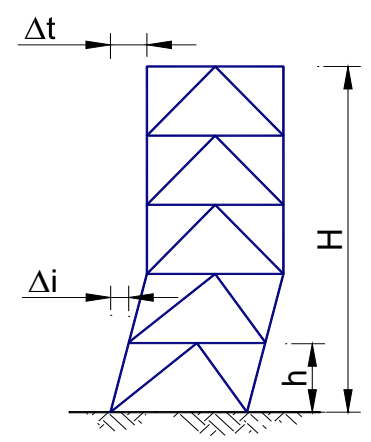

Figura 7. Criterios para establecer el factor de concentración por distorsión (DCF)

\section{Metodología propuesta por MacRae et al. (2004)}

MacRae et al. (2004) propusieron que el máximo factor de concentración por distorsión en marcos de acero contraventeados en la formación del mecanismo columna fuerte - viga débil - contraviento más débil $\left(D C F_{m}\right)$ se determine considerando el máximo valor obtenido de las ecuaciones 5 y 6 , a partir del número de niveles, la distribución vertical del cortante lateral resistente, la esbeltez de las columnas del primer nivel en relación con la rigidez lateral de los contravientos y la ductilidad esperada y supone que 
los contravientos desarrollan una fluencia elasto-plástica perfecta y que las columnas del primer nivel fluirán.

$D C F_{1}=1+\frac{7 \beta N^{2}}{120\left(\alpha+\beta N^{2} / 15\right)}$

$D C F_{2}=\frac{1+0.64(\mu-1)^{0.5}(1-\beta \prime / 3)\left(\beta^{\prime 0.319} / \alpha^{0.223}\right) N^{0.35}}{\mu^{0.5}\left(1-\beta^{\prime} / 3\right)}$

En las ecuaciones, $N$ es el número de niveles, $\alpha$ es una proporción de rigidez en el primer nivel (la suma de la rigidez flexionante de todas las columnas del primer nivel y la rigidez lateral del sistema de contravientos en ese nivel), $\mu$ es la ductilidad global de diseño, $\beta$ es un parámetro que describe la distribución de la resistencia lateral en la altura y $\beta$ ' es un parámetro que considera la distribución de la resistencia lateral con el número de pisos. $\beta$ y $\beta$ ' evalúan la distribución de la resistencia en la altura del edificio, debido a que usualmente la resistencia lateral no es constante en la altura, sino que disminuye conforme el cortante lateral actuante disminuye (considerando una distribución triangular inversa). $\beta$ ' se determina como sigue:

$\beta^{\prime}=(1-1.3 / N) \beta^{(1.25+(0.5 / N))}$

En la determinación del factor de concentración de distorsión se consideró que la ductilidad es igual a la ductilidad determinada en la metodología propuesta (ec. 2); la proporción $\alpha$ es igual a la aportación de las columnas en relación a los contravientos (ec. 1) y el parámetro $\beta$ se determinó considerando la carga lateral actuante y el cortante lateral resistente de diseño. Los factores de concentración se reportan en la tabla 2.

Tabla 2. Factores de concentración de distorsión según MacRae et al (2004)

\begin{tabular}{|c|c|c|c|c|c|}
\hline Modelos & $\begin{array}{c}\text { Proporción } \\
\alpha \\
\end{array}$ & $\begin{array}{c}\text { Parámetro } \\
\beta \\
\end{array}$ & $\begin{array}{c}\text { Parámetro } \\
\beta\end{array}$ & $\mathrm{DCF}_{1}$ & $\mathrm{DCF}_{2}$ \\
\hline Ch10_56 у Ch10_56a & 0.56 & 1.60 & 1.603 & 1.831 & 2.787 \\
\hline Ch14_61 y Ch14_61a & 0.61 & 1.56 & 1.607 & 2.992 & 1.849 \\
\hline Ch18_69 y Ch18_69a & 0.69 & 1.54 & 1.608 & 3.476 & 1.860 \\
\hline
\end{tabular}

\section{Metodología propuesta por Miranda (1999)}

Un segundo método disponible se publicó anteriormente en Miranda (1999), basado en la ductilidad global, el número de pisos y el modo de deformación que gobierna la estructura (por cortante o por flexión) mediante la ecuación 8 (Izvernari 2007).

$D C F_{3}=\beta_{2} \beta_{4} \frac{u_{a z}}{H}$

El factor $\beta_{2}$ depende del modo de deformación de la estructura, que se supuso igual a $\beta_{2}=1.6$ considerando valores intermedios recomendados en Izvernari (2007) y $u_{a z}$ es el desplazamiento máximo en la azotea determinado en un análisis elástico. Conforme al método propuesto, $\beta_{4}$ se determina con la ecuación 9, donde $\mu$ es la ductilidad esperada y $N$ el número de niveles. La magnitud de los factores obtenidos en este método se reporta en la tabla 3. 


$$
\beta_{4}=1+\frac{\mu}{30}+\frac{N}{200}
$$

Tabla 3. Factores de concentración según Miranda (1999)

\begin{tabular}{ccccc}
\hline Modelos & $\begin{array}{c}\text { Factor } \\
\beta_{2}\end{array}$ & $\begin{array}{c}\text { Factor } \\
\beta_{4}\end{array}$ & $\begin{array}{c}\text { Desplazamiento } \\
u_{a z}(\mathrm{~cm})\end{array}$ & \multirow{2}{*}{$\mathrm{DCF}_{3}$} \\
\hline Ch10_56 & 1.60 & 1.150 & 7.90 & 1.453 \\
Ch10_56a & 1.60 & 1.150 & 8.50 & 1.552 \\
Ch14_61 & 1.60 & 1.148 & 11.00 & 1.901 \\
Ch14_61a & 1.60 & 1.148 & 10.81 & 1.810 \\
Ch18_69 & 1.60 & 1.157 & 17.58 & 1.807 \\
Ch18_69a & 1.60 & 1.157 & 18.46 & 1.898 \\
\hline
\end{tabular}

En un estudio similar, Izvernari (2007) obtuvo DCFs iguales a 2.2, 2.9 y 5.6 para modelos de 8, 12 y 16 niveles respectivamente, estructurados con marcos de acero contraventeados en configuración en $\mathrm{X}$ con columnas de sección I y contravientos de sección cajón, mientras que MacRae et al. (2004) obtuvo DCFs iguales a 4.9, 6.3 y 8.1 en modelos de 10, 12 y 15 pisos con edificios regulares estructurados con base en marcos de acero con contravientos en cruz.

En la figura 8 se presenta el DCF en el 100 percentil obtenido en los análisis y se compara con los factores determinados con los métodos comentados. La magnitud de los DCFs obtenidos en este estudio son cercanos a la unidad, lo que debe interpretarse como una mejor tendencia a la distribución en la altura del comportamiento inelástico y que a su vez son aún menores a los factores de concentración esperados por las metodologías de predicción incluidos en la gráfica.

\section{Distorsiones obtenidas}

En la figura 9 se presentan las distorsiones de fluencia y las distorsiones últimas de cada entrepiso en relación al límite de las NTCS-04 igual a $\delta_{\text {servicio }}=0.4 \%$ para la revisión por limitación de servicio (no daño) e igual a $\delta_{\text {colapso }}=1.5 \%$ en la revisión para seguridad contra colapso (prevención de colapso). En la gráfica no se incluye la magnitud de la distorsión de los niveles que no presentaron fluencias, que fueron básicamente los pisos superiores y en algunos casos el nivel de planta baja, pero si se reporta la distorsión última alcanzada por todos los entrepisos.

Las distorsiones de fluencia son bastante cercanas al límite que proponen las NTCS-04, pese a que algunos entrepisos de los modelos que consideran la sobrerresistencia del material superan el $0.4 \%$ y otros fluyen a una distorsión menor. Las distorsiones últimas de los entrepisos intermedios de todos los modelos superan el límite establecido del 1.5\%. En general, la sobrerresistencia del material aumenta la magnitud de la distorsión de la primera fluencia de los entrepisos, pero no incrementa necesariamente la distorsión última.

El promedio de las distorsiones de entrepiso obtenidas en cada análisis se reportan en la tabla 4, sin considerar las magnitudes del primer entrepiso por su condición de apoyo. Los resultados de las distorsiones de fluencia denotan dependencia con la altura, de manera que, en conformidad con esta tendencia, el límite $\delta_{\text {yperm }}=0.40 \%$ se satisface en modelos de mediana y baja altura. Si este límite no queda satisfecho, significa que el daño inicial en los elementos estructurales se presentará después de la deformación proyectada desde la etapa elástica y se asociará al daño de elementos no estructurales. En cambio, si se satisface prematuramente (como en el modelo de 18 pisos), significa que el daño en la 
estructura se presentará antes de lo previsto. El promedio de las distorsiones reportadas es igual a $\delta_{y \text { prom }}=$ 0.379 , que es bastante similar al límite vigente.
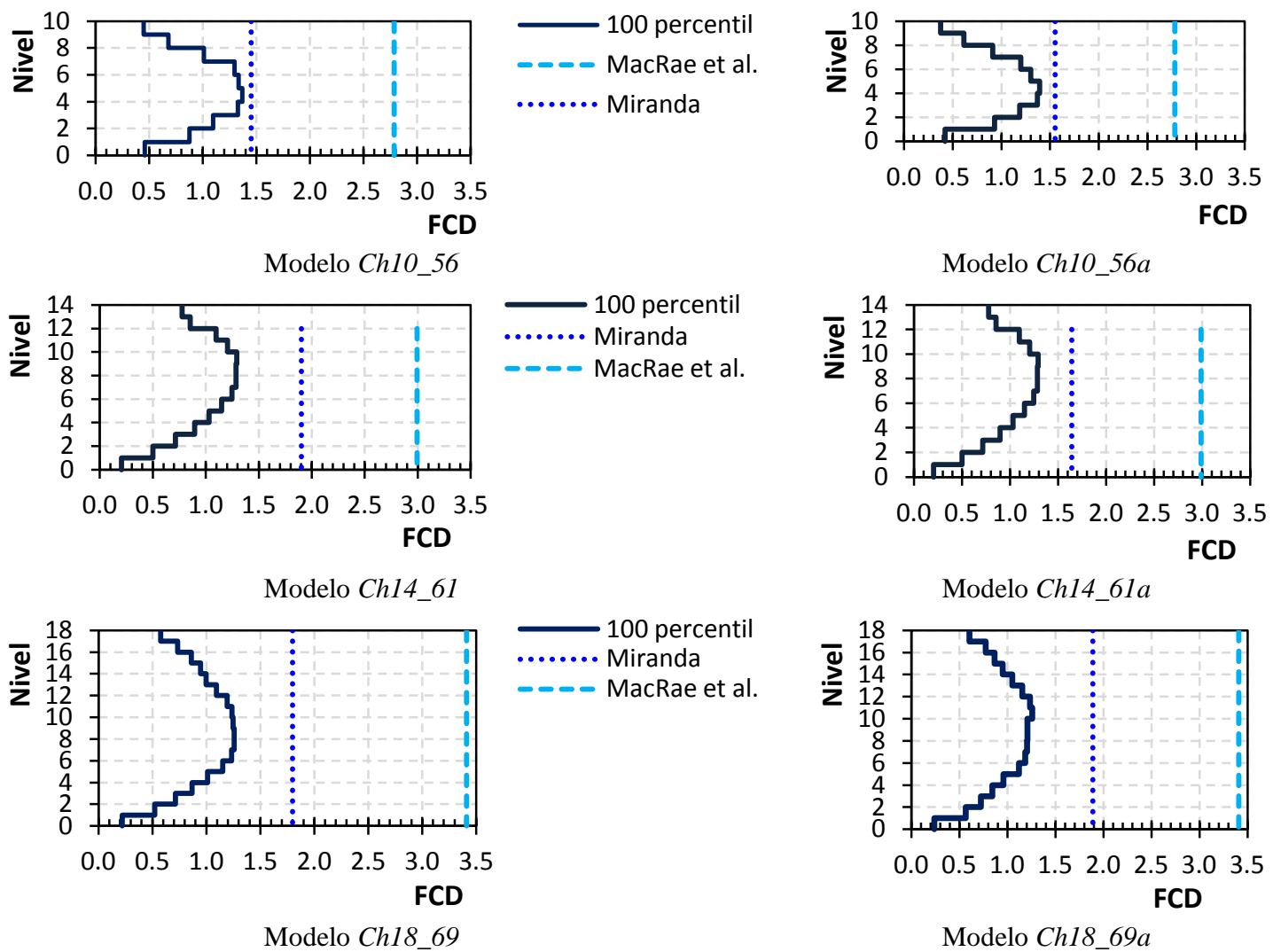

Figura 8. Distorsiones de diseño (\%) y su relación con el número de pisos de los modelos

La localización de las mayores deformaciones de fluencia concentradas en los pisos inferiores del modelo de 18 pisos hace notar, al parecer, una tendencia inicial de la estructura hacia la formación de un mecanismo de pisos débil. Sin embargo, el aumento de la resistencia de las columnas (ecuación 1) parece corregir la tendencia obligando a que los entrepisos superiores participen en la disipación de energía, logrando una mejor distribución de la participación inelástica en el mecanismo final. La tendencia a la formación de pisos débiles en estructuras con este sistema estructural no es atípica y ha sido identificado previamente en otras investigaciones (por ejemplo Tapia 2005, MacRae et al. 2004, Lacerte y Tremblay 2006) en edificios que no consideraban las propuestas de diseño presentadas en este articulo.

Por otra parte, no se observa alguna tendencia en el promedio de las distorsiones últimas reportadas con magnitudes que satisfacen el requisito impuesto. El promedio de las distorsiones últimas reportadas en la tabla 4 es $\delta_{u \text { prom }}=1.389$, que es menor al límite que proponen las NTCS ( $\delta_{\text {perm }}=1.5 \%$ ); sin embargo, será necesario aumentar la cantidad de modelos estudiados antes de proponer un ajuste al límite normativo. 

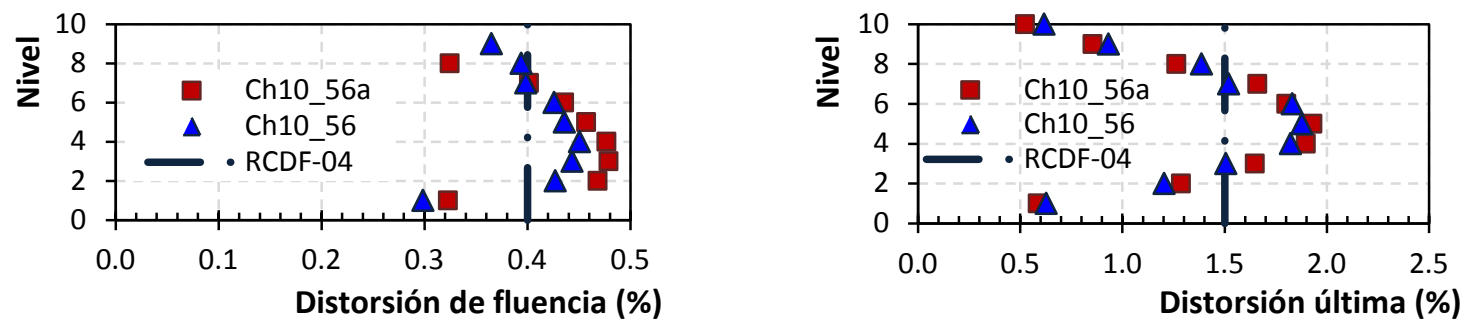

Modelos de 10 niveles
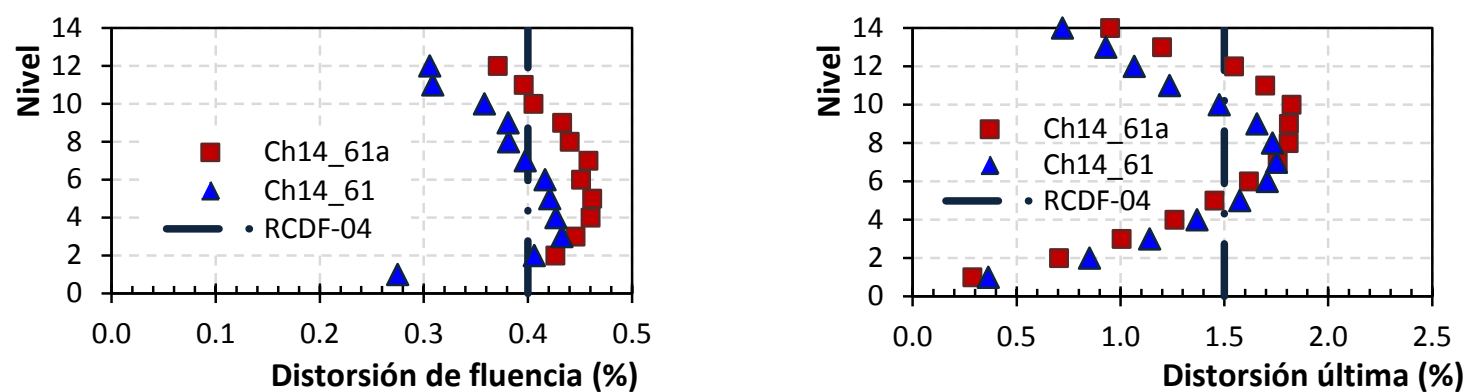

Modelos de 14 niveles
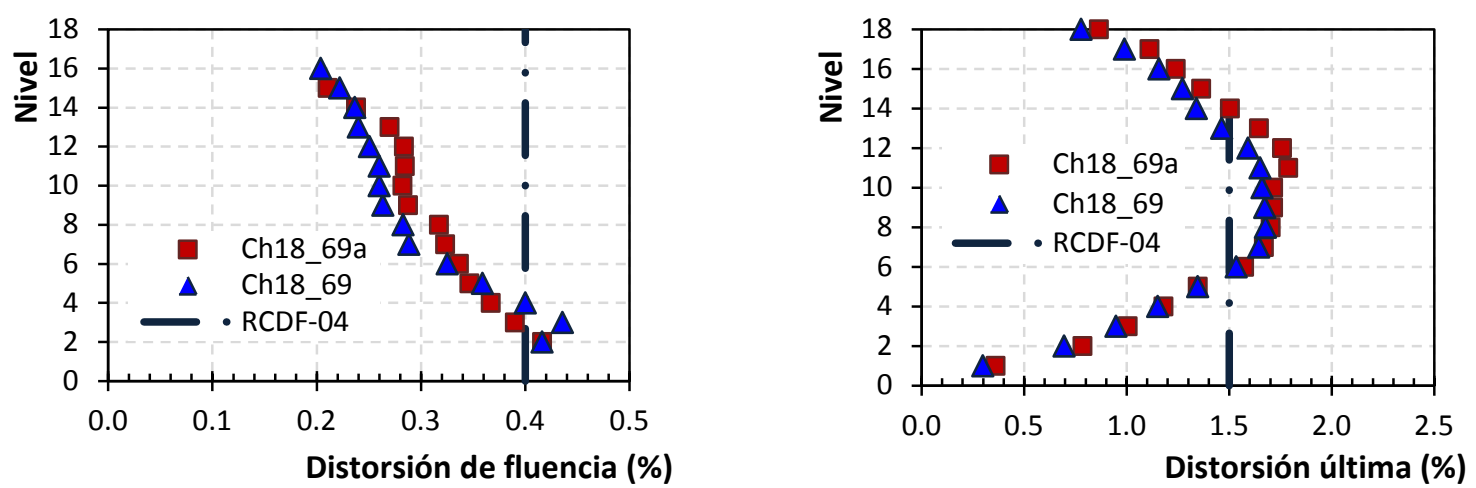

Modelos de 18 niveles

Figura 9. Envolventes de respuesta de deformación de los modelos de 10, 14 y 18 niveles

Tabla 4. Distorsiones desarrolladas por los modelos (\%)

\begin{tabular}{ccc}
\hline Modelo & $\begin{array}{c}\text { Distorsión de } \\
\text { fluencia }\end{array}$ & Distorsión última \\
\hline Ch10_56 & 0.417 & 1.410 \\
Ch10_56a & 0.435 & 1.428 \\
Ch14_61 & 0.385 & 1.324 \\
Ch14_61a & 0.432 & 1.433 \\
Ch18_69 & 0.296 & 1.328 \\
Ch18_69a & 0.311 & 1.409 \\
\hline
\end{tabular}

\section{Espectros de demanda de ductilidad}

Una vez determinada la capacidad sismo-resistente última de la estructura mediante un análisis estático no lineal y su periodo fundamental mediante un análisis modal espectral, fue posible identificar los acelerogramas para los que la estructura sería más demandada para una cierta demanda de ductilidad 
(Tena 1998, Tena-Colunga 2001). Por esta razón, a partir de las curvas globales cortante - distorsión (figura 10) fue posible calcular la rigidez inicial $\left(k_{1}\right)$ y la rigidez del modelo post - fluencia $\left(k_{2}\right)$, así como la proporción entre ambas $\left(k_{2} / k_{1}\right)$.
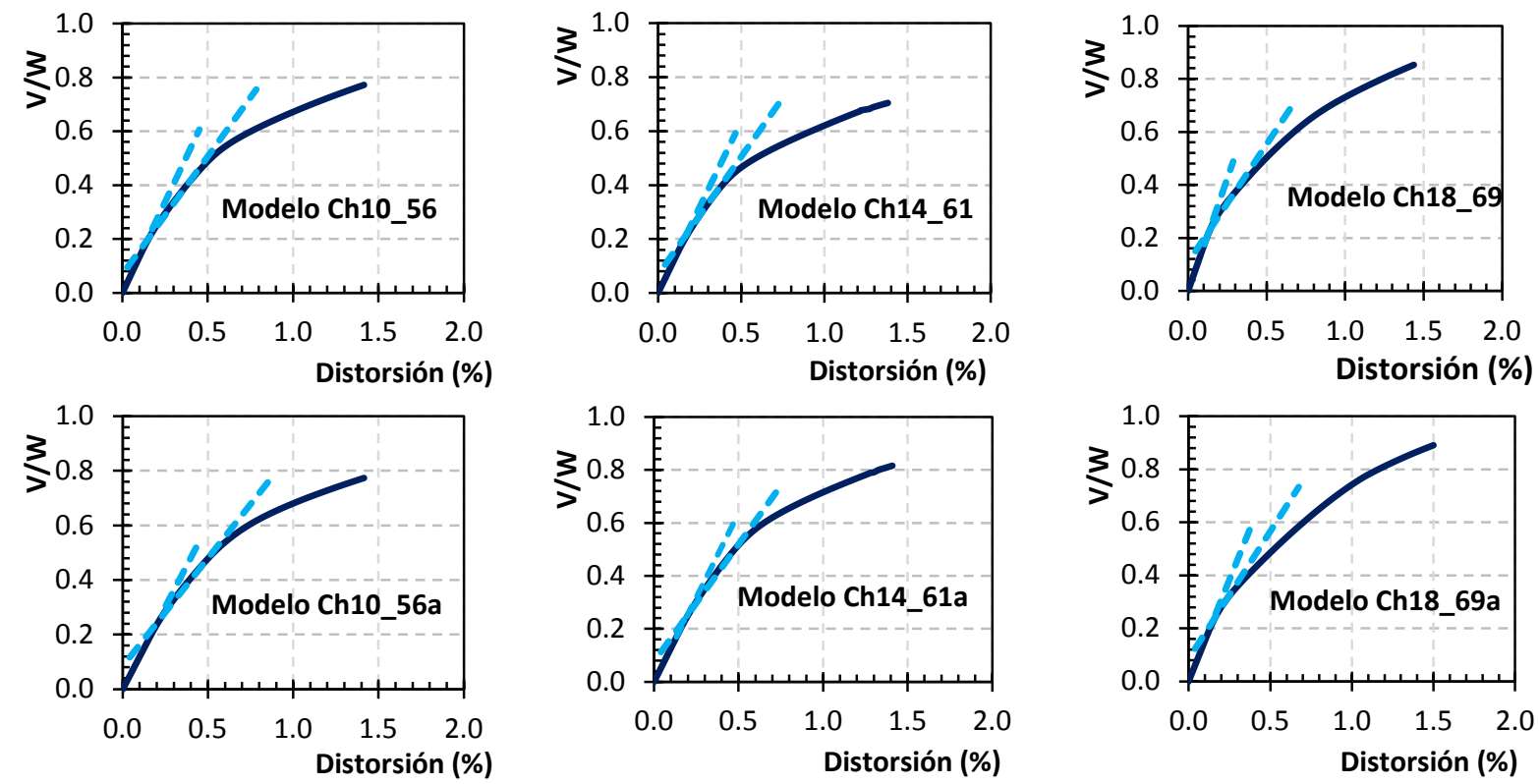

Figura 10. Curvas globales cortante - distorsión de los modelos

Los acelerogramas considerados fueron generados por Godínez (2010) para sismos de subducción con epicentros en la costa de Guerrero, a partir de estaciones acelerométricas ubicadas en la zona IIIa, de manera que para cada estación se realizaron simulaciones numéricas para obtener registros congruentes con el peligro sísmico de la zona estudiada. Los detalles del procedimiento empleado se puede consultar en Godínez (2010) y Godínez-Domínguez et al. (2012). Todos los registros considerados tienen una duración de $200 \mathrm{seg}$ con un intervalo de $0.02 \mathrm{seg}$, con excepción del registro s84, que fue generado por Pérez Rocha y que tiene una duración de 245.76 seg con un intervalo de 0.06 seg.

En la figura 11, se compara el espectro de respuesta elástico de cada registro seleccionado contra el espectro de diseño elástico calculado conforme al Apéndice A para un sitio con $\mathrm{T}_{\mathrm{s}}=1.2$ seg ubicado en la subzona IIIa, para demostrar que los acelerogramas sintéticos se ajustan razonablemente bien con el peligro sísmico considerado. En la tabla 5 se enuncian las aceleraciones máximas en cada dirección y la magnitud normalizada con la aceleración de la gravedad de los registros considerados.

Además, en la figura 12 se incluye el espectro de demanda de ductilidad del modelo Ch14_61a de 14 niveles con sobrerresistencia del material considerando los diez registros seleccionados para ilustrar el procedimiento empleado. Tapia (2011) presenta el resto de los espectros de demanda de ductilidad determinados para los modelos estudiados. 


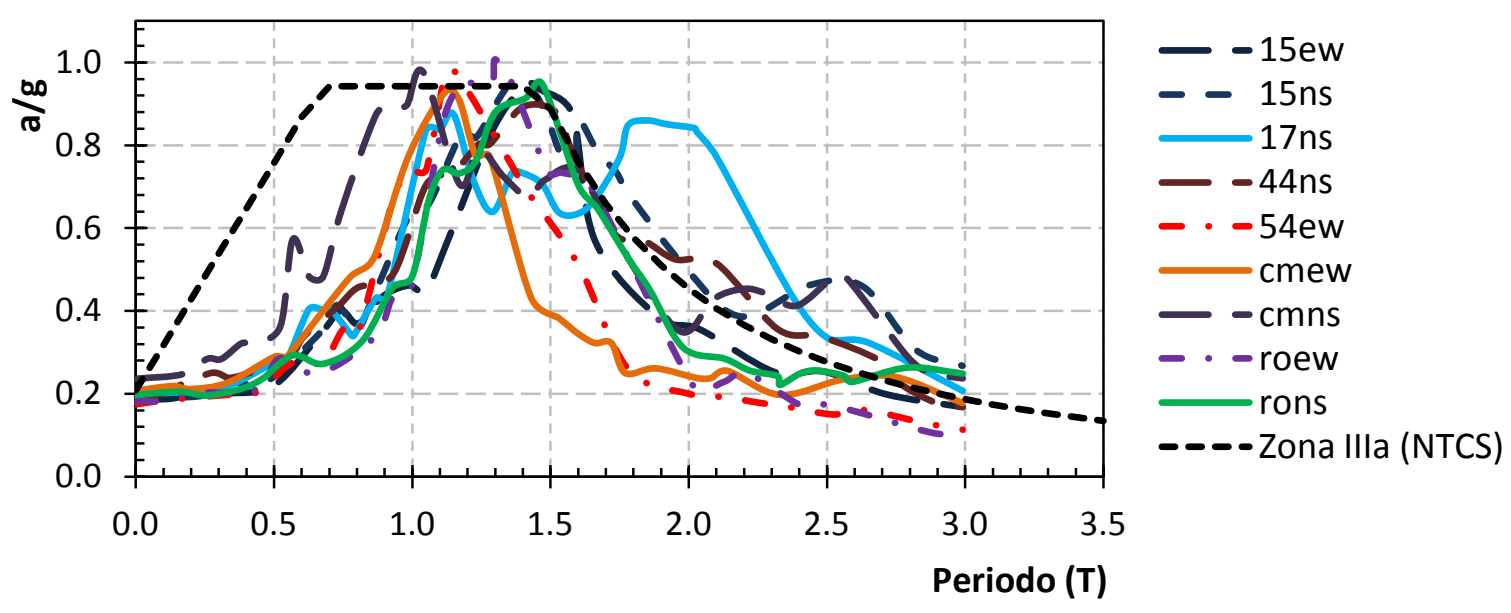

Figura 11. Espectros de respuesta y el espectro de diseño elástico

Tabla 5. Características de los acelerogramas seleccionados en este estudio

\begin{tabular}{ccccc}
\hline \multirow{2}{*}{ Registro } & \multicolumn{2}{c}{ Dirección norte - sur } & \multicolumn{2}{c}{ Dirección este - oeste } \\
\cline { 2 - 5 } & $\begin{array}{c}\text { Aceleración } \\
\text { máxima } S_{a \max } \\
\left(\mathrm{cm} / \mathrm{s}^{2}\right)\end{array}$ & $S_{a \max } / g$ & $\begin{array}{c}\text { Aceleración } \\
\text { máxima } S_{a \max }\end{array}$ & $S_{a \max } / g$ \\
$\left(\mathrm{~cm} / \mathrm{s}^{2}\right)$ & \\
\hline 15 & 183 & 0.947 & 185 & 0.951 \\
44 & - & - & 217 & 0.911 \\
$\mathrm{CM}$ & 203 & 0.929 & 232 & 0.977 \\
$\mathrm{RO}$ & 179 & 1.007 & 193 & 0.948 \\
54 & 171 & 0.973 & - & - \\
17 & - & - & 194 & 0.875 \\
\hline
\end{tabular}

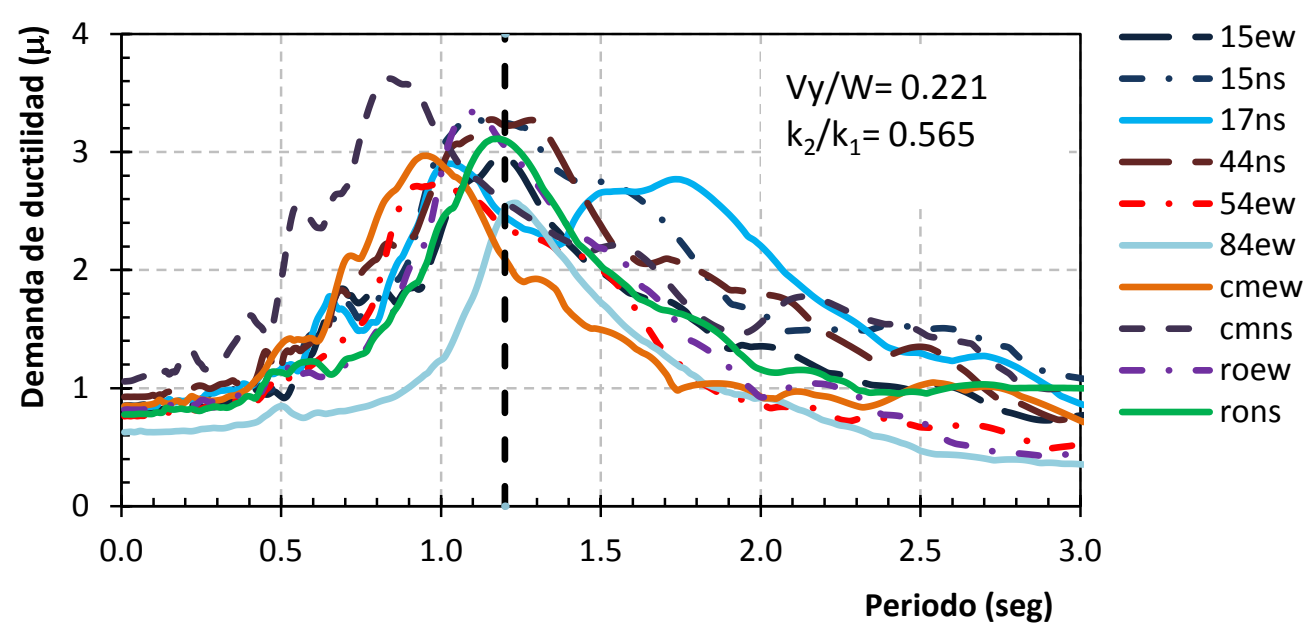

Figura 12. Espectros de demanda de ductilidad del modelo Ch14_61a 


\section{ANÁLISIS DINÁMICOS NO LINEALES}

Con la información obtenida de los análisis estáticos no lineales, se realizaron análisis dinámicos paso a paso en el programa OpenSees (Mazzoni et al 2006) para estudiar las demandas acumuladas de rotación plástica en trabes y columnas y acortamientos y alargamientos en contravientos a lo largo de los análisis. De esta manera fue posible identificar la acumulación del daño asociado a los registros que ocasionaron las mayores demandas en cada caso: estación CM en dirección norte - sur (registro cmns) en el modelo de 10 pisos, estación 44 en dirección norte - sur (registro 44ns) en el modelo de 14 pisos y en la estación 17 en dirección norte - sur (registro 17ns) en el modelo de 18 pisos. Las rotaciones plásticas máximas se compararon con la rotación máxima que pueden soportar los elementos realmente considerando los resultados de Kemp (1996), como se comentó anteriormente.

Los mapeos de las demandas acumuladas se normalizaron en cinco escalas de colores en función de la máxima rotación plástica en trabes y columnas y, por otra parte, el máximo alargamiento ó acortamiento presentado en los contravientos. Los casos incluidos en el artículo están asociados a las demandas máximas, pero no todos los registros tuvieron la misma magnitud de daño (Tapia 2011), lo que explica que los parámetros decrezcan si se considera el promedio de la respuesta. Asimismo, en cada edificio se determinó independientemente la rotación plástica, acortamiento o alargamiento máximos, de manera que no debe considerarse que la magnitud de las rotaciones sean equivalentes entre ellos.

Los modelos de 10 y 14 niveles, que fueron mayormente excitados en los análisis, presentan una buena distribución de la respuesta inelástica en la altura, con poco o nulo daño en la base de las columnas del marco, siendo el sistema de contravientos el que registra una mayor concentración de daño. Para ejemplificar lo anterior, en la figura 13 se presentan las demandas acumuladas del modelo de 14 niveles.

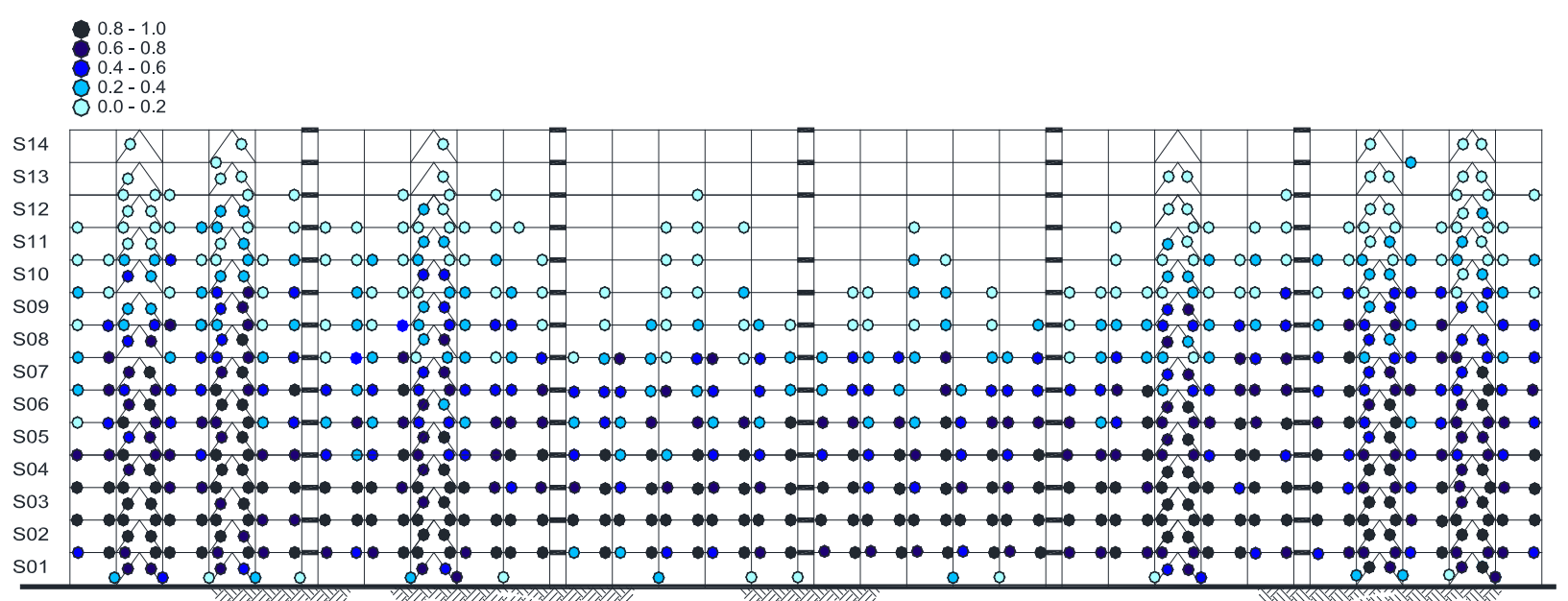

Figura 13. Mapeo de demandas acumuladas en Ch14_61a bajo el acelerograma 44ns

Vale la pena señalar que los modelos diseñados con los esfuerzos de fluencia teóricos (Ch10_56, Ch14_61 y Ch18_69) tienen una mayor cantidad de elementos con comportamiento inelástico y de mayor magnitud. Sin embargo, sus resultados, que no consideran las fuentes de sobrerresistencia del material, no resultan los más representativos del comportamiento esperado de una estructura fabricada con los perfiles y placas comerciales disponibles en la Ciudad de México con un adecuado control de calidad y supervisión de obra. Por esta razón, no se incluyen ni se consideran para establecer conclusiones en esta investigación. 


\section{Distorsión asociada a la primera fluencia}

Se determinó la primera fluencia en el paso cuando un elemento estructural del entrepiso fluía asociado a las rotaciones superiores a la rotación elástica en trabes y columnas o en la deformación axial relacionada a la carga de pandeo en contravientos para evaluar si el límite propuesto en el reglamento es adecuado.

Conforme al Apéndice A (NTCS-04), la rigidez lateral de la estructura se evalúa revisando que la distorsión de entrepiso de los análisis (elásticos) multiplicada por el factor $Q$ ' $R / 7$ sea menor a $0.4 \%$ cuando los elementos incapaces de soportar deformaciones apreciables están separados de la estructura principal, sin considerar el tipo de estructuración ó el uso de la edificación que se está evaluando.

En la figura 14 se presenta el promedio de la distorsión en porcentaje de los entrepisos en el instante en el que fluye alguno de sus elementos de los análisis realizados, la magnitud máxima de la distorsión que se presentó en el entrepiso en alguno de los diez análisis y la distorsión de diseño obtenida de los análisis elásticos. Cuando algún entrepiso en la gráfica no reporta ninguna fluencia, implica que el entrepiso se comportó elásticamente en todos los análisis realizados.
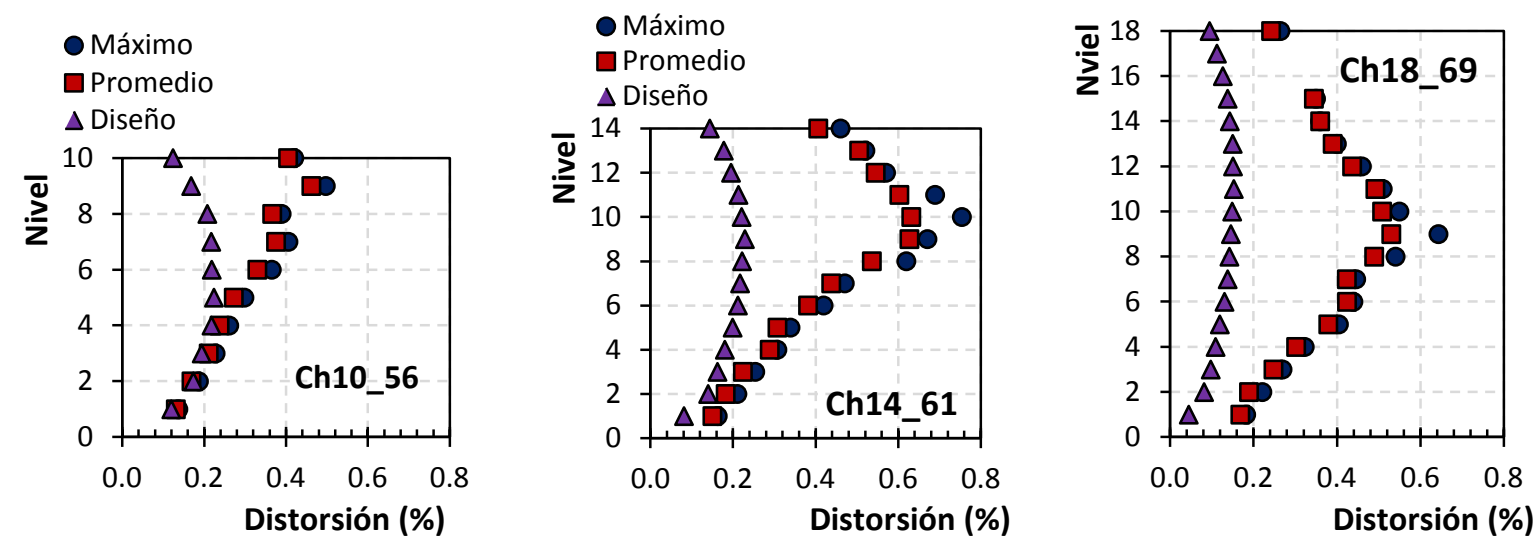

Figura 14. Envolvente de respuesta al inicio de la fluencia en los análisis realizados

En todos los casos la distorsión de diseño, que constituye el límite inferior, se aproxima bien a los resultados de los análisis en los pisos inferiores de los modelos, pero esta predicción favorable se pierde con el aumento en la altura de los niveles.

En los diez análisis realizados a cada uno de los tres modelos (Ch10_56a, Ch14_61a y Ch18_69a), se presentaron 314 entrepisos con comportamiento no lineal en total, siendo que el promedio de la distorsión a la primera fluencia de los entrepisos total es $\delta_{y}$ prom $=0.37 \%$, de manera que los resultados de este estudio coinciden aceptablemente bien con el límite propuesto en las NTCS-04 igual a 0.40\% en la revisión del estado límite de servicio.

\section{Distorsión máxima desde el origen}

En la figura 15 se incluye el comportamiento de las envolventes de respuesta de las distorsiones máximas en porcentaje medidas desde el origen, que incluye el límite propuesto en las NTCS-04 y la distorsión de diseño. Los resultados de los análisis de los modelos de 18 niveles, situados en la rama 
descendente de los espectros de demanda de ductilidad reportan magnitudes de deformación con una dispersión baja. Sin embargo, la disparidad de las magnitudes crece con el aumento de la demanda, como ocurre en los pisos inferiores de algunos análisis en los edificios de 10 y 14 pisos. En general, las distorsiones de diseño envuelven aceptablemente las distorsiones dinámicas máxima promedio (de 10 registros) en todos los modelos.

Note que la diferencia entre la distorsión de fluencia de diseño y la obtenida en los análisis no lineales aumenta con el incremento de la altura del modelo (figura 14) y que esta tendencia cambia si se estudian las distorsiones últimas (figura 15), es decir, hacia el final del análisis los modelos de mayor altura desarrollan deformaciones más controladas que los modelos altos (figura 15), tal que la distorsión última máxima de diseño del modelo de 18 pisos es significativamente menor al límite establecido en las NTCS. En caso que se hubiera mantenido la tendencia inicial de la distorsión de fluencia, sería válido sugerir que los modelos desarrollarían un mecanismo de piso débil, sobre todo en edificios altos. Sin embargo, los resultados de los modelos estudiados, mediante la aplicación de la metodología propuesta en esta investigación, sugieren que la deformación logra ser controlada mediante una mejor distribución de la respuesta inelástica en la altura hacia el final de los análisis (figura 15).
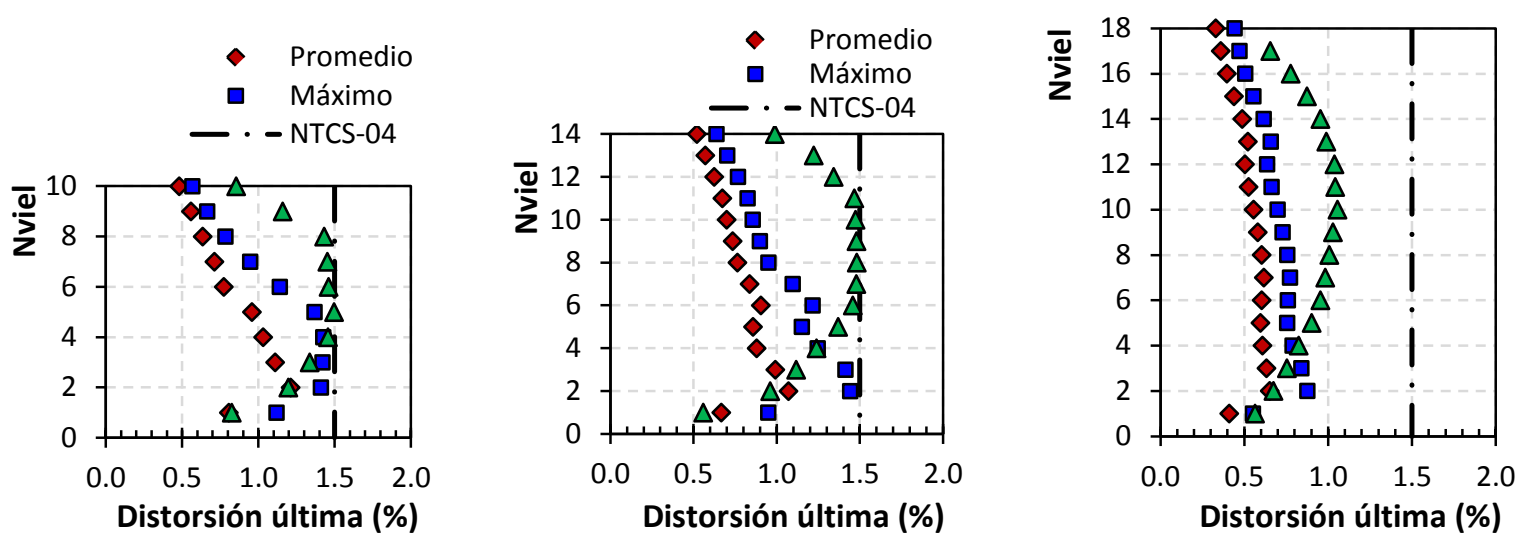

Figura 15. Envolventes de respuesta de las distorsiones máximas (\%) medidas desde el origen

Las respuestas máximas superan la distorsión esperada en la etapa de diseño en los pisos inferiores, de manera muy notable en los primeros tres niveles. En ningún caso las distorsiones máximas (medidas desde un estado de desplazamiento cero) son mayores al límite propuesto de las Normas Técnicas. La distorsión máxima de entrepiso alcanzada se presentó en el segundo piso del modelo de 14 niveles con una magnitud de $1.44 \%$ que sigue siendo menor al $1.50 \%$, por lo que el límite parecería adecuado para esta revisión.

\section{Distorsión máxima asociada al medio ciclo de histéresis}

Las magnitudes reportadas en el apartado inmediato anterior consideran la distorsión máxima que se alcanzó en los análisis a partir del origen sin considerar el efecto de asimetrías y/o deformaciones permanentes que provoca la solicitación dinámica cíclica; es decir, esa deformación considera la excitación referida al origen sin tomar en cuenta la energía total necesaria para llevar la estructura desde una fuerza nula hasta su máxima incursión inelástica en el otro extremo.

Por esta razón, en este estudio se incluyó la distorsión de entrepiso máxima $\delta_{\max }$ ilustrada en la figura 16; que mide la magnitud del desplazamiento desde la posición donde comienza a cargar el medio 
ciclo de máxima amplitud (que no implica que deba ser la posición inicial con desplazamiento cero) hasta el extremo opuesto. Los resultados de los modelos estudiados se muestran en la figura 17, donde se muestra el promedio de las distorsiones máximas de los diez análisis no lineales realizados (en rombos color rojo), la respuesta máxima obtenida (en rectángulos color azul) y el límite propuesto en las NTCS igual a $1.5 \%$.

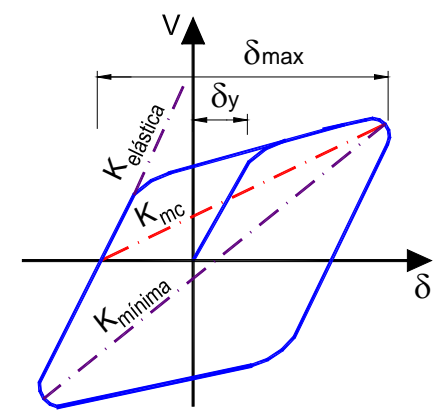

Figura 16. Definición de parámetros en ciclos inelásticos

En las gráficas se aprecia que las distorsiones reportan una magnitud significativamente más grande que las que se obtendría y considera para evaluar este requisito a partir del origen (desplazamiento cero), de manera que el límite de deformación (1.5\%) es superado ampliamente en los pisos intermedios de los modelos de 10 y 14 niveles.

Por lo anterior, cabe señalar que, aunque el requisito establecido en las NTCS debe compararse con la deformación generada desde un punto inicial con desplazamiento cero, esto no debe interpretarse como que esa distorsión será la máxima a la que estará sujeto el edificio.

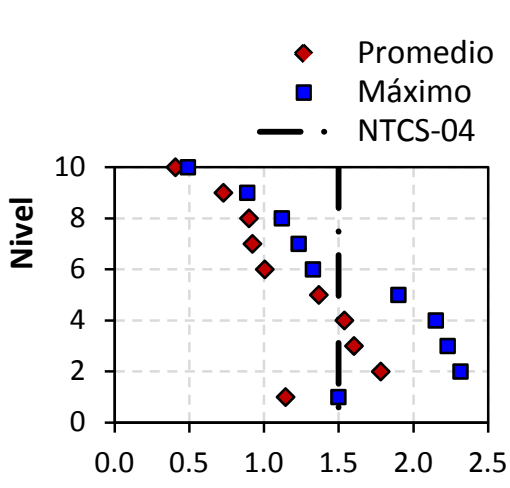

Distorsión máxima(\%), $\delta_{\max }$

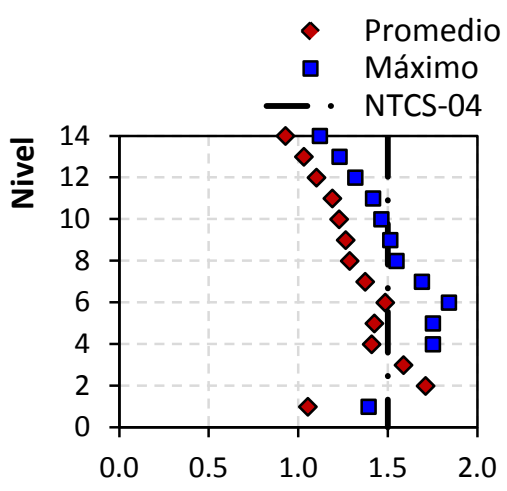

Distorsión máxima $(\%), \delta_{\max }$

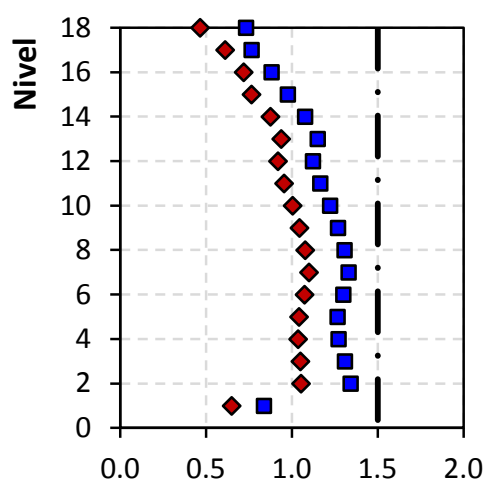

Distorsión máxima $(\%), \delta_{\max }$

Figura 17. Envolventes de respuesta de la distorsión máxima asociada al medio ciclo histerético

\section{Distorsiones residuales}

En Canadá y Estados Unidos es común diseñar este tipo de estructuras distinguiendo los marcos sismo-resistentes, usualmente situados en la periferia, de los marcos que soportarán únicamente cargas gravitacionales, siendo que en México es común que los edificios en zonas sísmicas sean diseñados con base en marcos momento - resistentes, donde todos los marcos aportan a la rigidez lateral del edificio. 
Entre otras cosas, este concepto de diseño ofrece una mayor estabilidad y control de daño ante sismos intensos como se discute en esta sección.

En la figura 18a se presentan las envolventes de las distorsiones residuales globales, es decir, las deformaciones permanentes después de efectuados los análisis dinámicos en función de la cantidad de pisos de los modelos, mientras que en la figura 20b se reportan las distorsiones máximas de entrepiso. Como se aprecia, aún en la mayor solicitación (distorsión máxima), los resultados las distorsiones permanentes son menores al $0.2 \%$, pese a que los modelos presentan daño significativo en los elementos que lo conforman (figura 13).

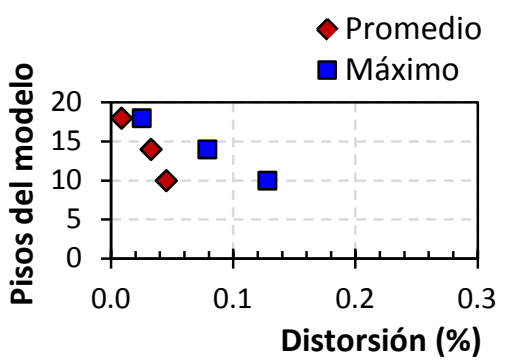

a) Deformaciones globales

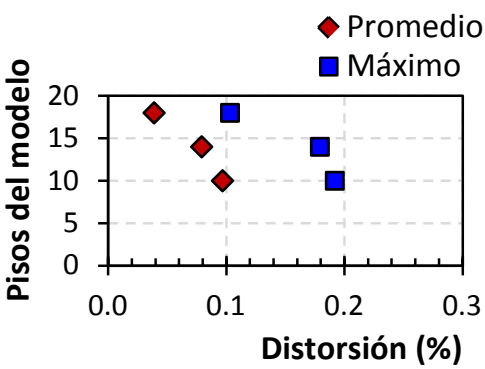

b) Deformaciones de entrepiso

Figura 18. Envolvente de distorsiones residuales

\section{DUCTILIDADES}

Con base en las curvas de histéresis, se relacionó el comportamiento no lineal de los modelos contra el factor de comportamiento sísmico propuesto en la etapa de diseño. Las magnitudes reportadas corresponden a la máxima ductilidad desarrollada en valor absoluto determinadas con la máxima deformación positiva o negativa. En la figura 19 se presenta las envolventes de respuesta de las curvas de histéresis de los entrepisos (deformación del entrepiso entre su altura contra el cortante de entrepiso) y considerando los resultados de la curva global (deformación en la azotea entre la altura total contra el cortante basal). En ella, no se reportan los entrepisos que presentaron un comportamiento lineal con una ductilidad igual a uno. En la figura se nota una disminución de la ductilidad con el aumento de la altura de los modelos que coincide con los resultados presentados en ésta y otras investigaciones (Izvernari 2007, Tapia y Tena 2009, Godínez 2010).

La mayor dispersión de ductilidades se presenta en el modelo de 10 niveles y demuestra que este edificio puede desarrollar deformaciones significativamente altas $\mathrm{y}$, por consiguiente, ductilidades grandes (superiores a 6 en algunos casos), lo que no sucede en los modelos de 14 y 18 pisos. Estos resultados son congruentes con el planteamiento de la ecuación 2, que relaciona la estimación de la ductilidad con la altura de los edificios.

Igualmente, la ductilidad de entrepiso máxima se presenta en el segundo nivel del modelo de 10 pisos (Ch10_56a) con una magnitud de $\mu_{\max }=6.09 \mathrm{y}$ un promedio de $\mu_{\text {prom }}=4.08$. Las curvas presentadas incluyen el factor de comportamiento sísmico $Q$ determinado con la metodología propuesta en esta investigación en función de la relación de esbeltez (tabla 1 obtenidos con la ecuación 2). 

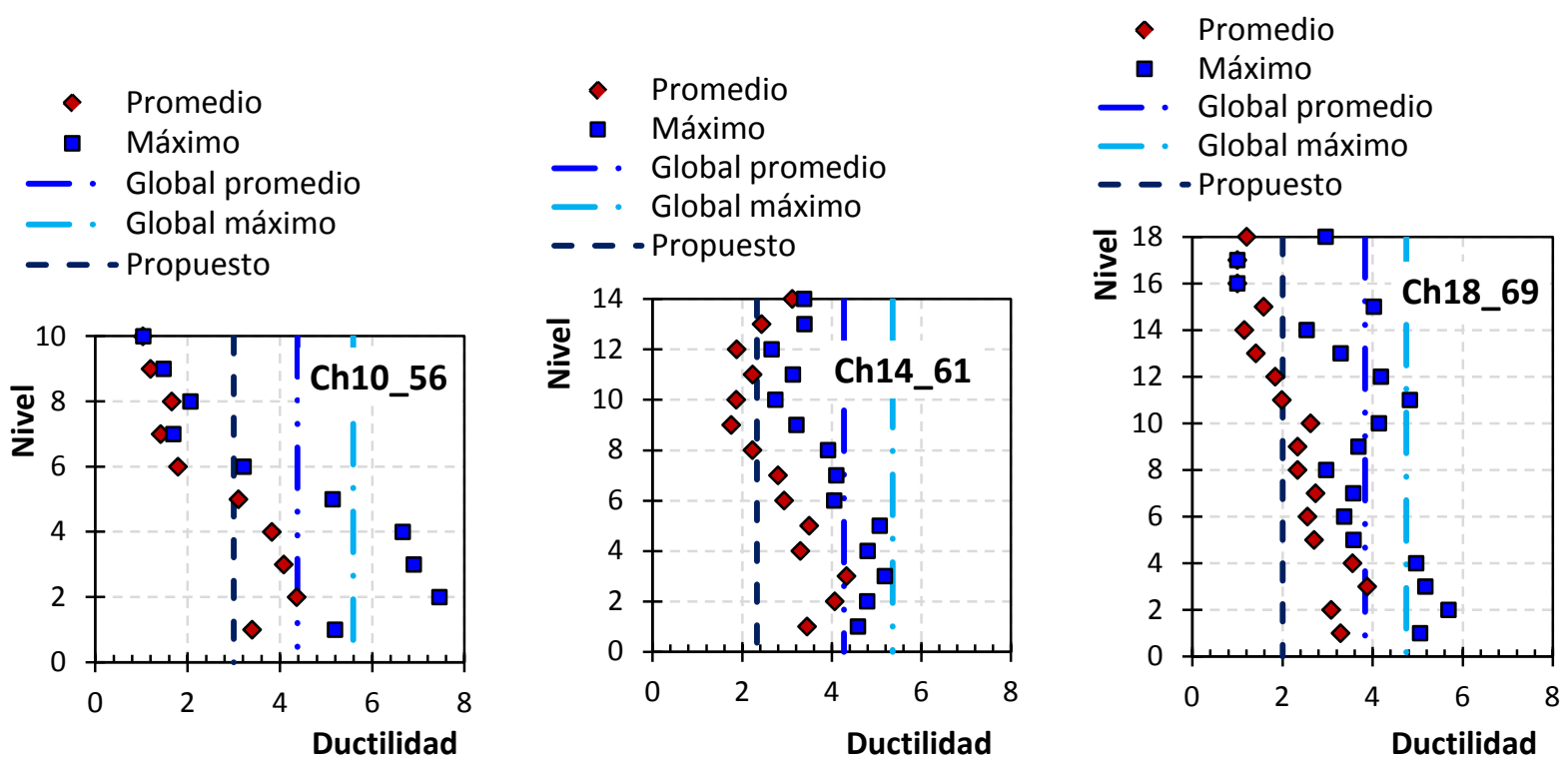

Figura 19. Demandas máximas de ductilidad de entrepisos y globales

En la tabla 6 se reporta el promedio de las ductilidades, que denota un comportamiento dependiente de la altura. El factor de reducción propuesto (ecuación 2), aunque no predice con entera exactitud la magnitud alcanzada en el promedio de los análisis, sí logra predecir con mayor certidumbre el comportamiento no lineal de la estructura.

Tabla 6. Comparación de las ductilidades obtenidas

\begin{tabular}{cccc}
\hline Modelo & $\begin{array}{c}\text { Relación de } \\
\text { esbeltez del } \\
\text { edificio, H/B }\end{array}$ & $\begin{array}{c}\text { Factor de } \\
\text { comportamiento } \\
\text { sísmico propuesto, } Q\end{array}$ & $\begin{array}{c}\text { Promedio de la } \\
\text { ductilidad, } \mu\end{array}$ \\
\hline Ch10_56a & 1.00 & 3.00 & 2.86 \\
Ch14_61a & 1.40 & 2.33 & 2.70 \\
Ch18_69a & 1.80 & 2.00 & 2.24 \\
\hline
\end{tabular}

\section{CONCLUSIONES}

En la investigación sintetizada en este artículo se realizaron análisis no lineales en OpenSees a edificios regulares de acero con contraventeo concéntrico de 10, 14 y 18 niveles con configuraciones y perfiles comúnmente usados en el Distrito Federal. Los modelos se diseñaron siguiendo un procedimiento de diseño por capacidad adaptado al RCDF-04, que pretende tener una mayor distribución de las fluencias de los elementos en la altura asociada al mecanismo de falla columna fuerte - viga débil - contraviento más débil, que se explica y ejemplifica detalladamente en el artículo complementario (Tapia y Tena 2013).

Además, a través de 289 certificados de laboratorio de aceros A-36 y A-572 Gr. 50 de espesores de placas entre $3 / 16$ ” $(0.476 \mathrm{~cm})$ a 2.5 ” $(6.35 \mathrm{~cm})$, se establecieron magnitudes realistas de su esfuerzo de fluencia real promedio, tal que para cada altura se realizaron análisis no lineales a dos grupos de modelos: uno considerando los esfuerzos teóricos y otro con los esfuerzos con la sobrerresistencia del material establecida de estas pruebas. 
Las distorsiones de entrepiso obtenidas en los análisis no lineales, a la mitad del análisis (50 percentil), a la mitad más una desviación estándar (84 percentil) y al final del análisis (100 percentil), y los factores de concentración de distorsión se estudiaron y compararon con los planteamientos en otras investigaciones, estableciendo que los modelos diseñados con la metodología propuesta son menos propensos a desarrollar pisos débiles, lo que posteriormente se confirmó mediante análisis paso a paso no lineales ante acelerogramas sintéticos asociados al espectro de diseño correspondiente.

Considerando el concepto de espectros de demandas de ductilidad, se seleccionaron diez acelerogramas artificiales congruentes con el espectro de diseño elástico para un sitio ubicado en la zona IIIa con $\mathrm{T}_{\mathrm{s}}=1.2 \mathrm{seg}$, según la metodología del Apéndice A, de manera que fue posible establecer los registros que causarán las mayores demandas sísmicas a las estructuras en los análisis dinámicos no lineales. De los resultados, el mapeo de las rotaciones plásticas en trabes y columnas y los acortamientos y alargamientos en contravientos, coinciden aceptablemente bien con la formación de un mecanismo de final columna fuerte - viga débil - contraviento más débil, con una buena distribución en la altura de la respuesta no lineal y poco o nulo daño en las columnas.

El promedio de las distorsiones al inicio del comportamiento inelástico de los 314 entrepisos que fluyeron en total es de $0.37 \%$, que coincide aceptablemente bien con la magnitud propuesta en las NTCS para la limitación de daños (0.40\%). Adicionalmente, las distorsiones máximas se estudiaron en dos sentidos: la distorsión máxima medida a partir del origen (desplazamiento cero) y la distorsión máxima asociada la excitación dinámica cíclica, es decir, desde una fuerza nula en un punto extremo hasta su máxima incursión inelástica en el lado opuesto. La revisión de deformación por seguridad contra colapso (1.5\%) se satisface aún en los modelos que presentaron las distorsiones más grandes, donde la demanda máxima de diseño es la envolvente de las magnitudes reportadas en casi todos los entrepisos.

Finalmente, el promedio de las ductilidades de entrepiso en el modelo de 10 niveles resultó ser igual a 2.86, mientras que en los modelos de 14 y 18 pisos fue igual a 2.70 y 2.24, respectivamente. Los resultados confirman la dependencia con la altura que se había identificado con anterioridad y son cercanos a las magnitudes determinadas con la metodología propuesta en esta investigación $\left(Q_{10}=3.0\right.$ en el modelo de 10 niveles, igual a $Q_{14}=2.33$ en el modelo de 14 niveles e igual a $Q_{18}=2.0$ en el modelo de 18 pisos).

De acuerdo con estos resultados, los edificios estructurados con marcos con contraventeo concéntrico diseñados racionalmente con la metodología propuesta, desarrollan una adecuada distribución de la respuesta inelástica en la altura y un mecanismo de colapso congruente con las premisas iniciales de diseño (columna fuerte - viga débil - contraviento más débil), que permite a los diseñadores predecir con mayor certidumbre la ductilidad global y sobrerresistencia que el sistema puede desarrollar.

\section{REFERENCIAS}

ATC-63 (2008), “Quantification of buildings seismic performance factors”, ATC-63 Project Report - 90\% Draft. FEMA P695, abril.

Godínez, E (2010), "Estudio del comportamiento de marcos dúctiles de concreto reforzado con contraventeo chevrón”, Tesis de Doctorado, Posgrado en Ingeniería Estructural, División de Ciencias Básicas e Ingeniería, Universidad Autónoma Metropolitana Azcapotzalco, julio.

Godínez-Domínguez, E A, A Tena-Colunga y L E Pérez-Rocha (2012), "Case studies on the seismic behavior of reinforced concrete chevron braced framed buildings”, Engineering Structures, Vol. 45, No. 12, pp. 78-103, diciembre, DOI http://dx.doi.org/10.1016/j.engstruct.2012.05.005. 
Izvernari, C (2007), “The seismic behavior of steel braces with large sections”, Tesis de maestría, Département des génies civil, géologique et mines, École Polytechnique de Montréal, Canadá, abril.

Kemp, A (1996), "Inelastic local and lateral buckling in design codes”, ASCE Journal of Structural Engineering, Vol. 122, No. 4, pp. 374-382, abril.

Lacerte, M y R Tremblay (2006), "Making use of brace overstrength to improve the seismic response of multistory split-X concentrically braced steel frames”, Canadian Journal of Civil Engineering, Vol. 33, pp. 1005-1021.

MacRae, G, Y Kimura y C Roeder (2004), "Effect of column stiffness on braced frame seismic behavior”, Journal of Structural Engineering, ASCE, Vol. 130, No. 3, pp. 381-391, marzo.

Mazzoni, S, F McKenna, M Scott y G Fenves (2006), “Open system for earthquake engineering simulation, user command-language manual”, Report NEES grid-TR 2004-21. Pacific Earthquake Engineering Research, University of California. Berkeley, CA. http://opensees.berkeley.edu.

Ozgur, B (2006), "Response modification factor for concentrically braced steel frames". Tesis de maestría, Universidad Bogazici, Turquía.

NTCS-04 (2004), “Normas técnicas complementarias para diseño por sismo”, Gaceta Oficial del Distrito Federal, décimo cuarta época, tomo II, octubre.

RCDF-04 (2004), "Reglamento de Construcciones para el Distrito Federal (RCDF)”, Gaceta Oficial del Distrito Federal, décima cuarta época, enero.

Tapia, E (2005), "Estudio de los criterios del RCDF-2004 y sus normas técnicas complementarias para el diseño sísmico de edificios regulares con base en marcos de acero", Tesis de Maestría, División de Estudios de Posgrado de la Facultad de Ingeniería de la Universidad Nacional Autónoma de México, abril.

Tapia, E (2011), “Comportamiento de edificios regulares estructurados con marcos dúctiles de acero con contraventeo concéntrico en suelo blando". Tesis de doctorado, Posgrado en Ingeniería Estructural, División de Ciencias Básicas e Ingeniería, Universidad Autónoma Metropolitana Azcapotzalco, julio.

Tapia, E y A Tena (2007), "Estudio del comportamiento de marcos dúctiles de acero con contraventeo concéntrico en edificios regulares en zonas sísmicas”, Memorias, XVI Congreso Nacional de Ingeniería Sísmica, Ixtapa, Guerrero, noviembre.

Tapia, E y A Tena (2009), "Factores de ductilidad y sobrerresistencia en marcos de acero con contraventeo concéntrico”. Memorias, XVII Congreso Nacional de Ingeniería Sísmica, Puebla, Puebla, noviembre.

Tapia, E y A Tena (2011), "Factores de ductilidad y sobrerresistencia en marcos de acero con contraventeo chevrón”, Revista de Ingeniería Sísmica, SMIS, No. 84, pp. 46-68.

Tapia, E y A Tena (2013), "Diseño sísmico de marcos de acero contraventeados. Parte 1: Recomendaciones de diseño”, Revista de Ingeniería Sísmica, SMIS, No. 88, pp. 43-68.

Tena, A (1998), "Evaluación sísmica simplificada de estructuras existentes”, Revista de Ingeniería Sísmica, SMIS, No. 59, pp. 1-36.

Tena-Colunga, A. (2001), "Displacement ductility demand spectra for the seismic evaluation of structures”, Engineering Structures. Vol. 23, febrero, pp. 1319-1330. 